\title{
BODE'S LAW
}

\author{
M. LECAR
}

Center for Astrophysics, Cambridge, Mass. 02138, U.S.A.

M. W. Ovenden (Nature 239 (1972) 508) has outlined a theory intended to provide a dynamical explanation for Bode's law. The author suggests instead that the approximately constant spacing ratio expressed in Bode's mnemonic can be generated by a sequence of random numbers subject to the constraint that adjacent planets cannot be too close to each other.

The full text of the paper appeared in Nature 242 (1973) 318.

\section{DISCUSSION}

R. H. Miller: You should be able to get almost any distribution you want by choosing the radial distribution of gas mass (the initial angular momentum distribution) of the right form.

S. F. Dermott: I have recently analyzed the statistical significance of Bode's law (Nature 244 (1973) 17) and found that if it is allowed that ratios of adjacent orbital periods are not too close to unity then it is not too difficult to fit random periods into a Bode-type law.

D. C. Heggie: If it were true that Bode's law, obtained by plotting $\log a$, were accidental, surely one should obtain equally successful Bode's laws by plotting $a$ itself.

S. F. Dermott: It is not disputed that the planetary orbits are nonrandom on a linear scale. Bode's law effectively states that the orbits are nonrandom on a logarithmic scale. This is a far more interesting postulate, but I consider it to be untrue. 